\title{
New constraints on the 3-3-1 model with right-handed neutrinos
}

\author{
B. L. Sánchez-Vega ${ }^{1, \mathrm{a}}$, E. R. Schmitz ${ }^{2, \mathrm{~b}}$, J. C. Montero ${ }^{1, \mathrm{c}}$ \\ ${ }^{1}$ Instituto de Física Teórica (IFT), Universidade Estadual Paulista (Unesp), R. Dr. Bento Teobaldo Ferraz 271, Barra Funda, São Paulo, \\ SP 01140-070, Brazil \\ ${ }^{2}$ Bethe Center for Theoretical Physics and Physikalisches Institut, Universitaet Bonn, Nussallee 12, 53115 Bonn, Germany
}

Received: 7 August 2017 / Accepted: 10 February 2018 / Published online: 24 February 2018

(C) The Author(s) 2018. This article is an open access publication

\begin{abstract}
In the framework of a 3-3-1 model with righthanded neutrinos and three scalar triplets we consider different spontaneous symmetry breaking patterns seeking for a non-linear realization of accidental symmetries of the model, which will produce physical Nambu-Goldstone (NG) bosons in the neutral scalar spectrum. We make a detailed study of the safety of the model concerning the NG boson emission in energy-loss processes which could affect the standard evolution of astrophysical objects. We consider the model with $\mathrm{a} \mathbb{Z}_{2}$ symmetry, conventionally used in the literature, finding that in all of the symmetry breaking patterns the model is excluded. Additionally, looking for solutions for that problem, we introduce soft $\mathbb{Z}_{2}$-breaking terms in the scalar potential in order to remove the extra accidental symmetries and at the same time maintain the model as simple as possible. We find that there is only one soft $\mathbb{Z}_{2}$-breaking term that enables us to get rid of the problematic NG bosons.
\end{abstract}

\section{Introduction}

Recently experiments have reached the capability of exploring the TeV energy scale and the Standard Model (SM) still has an impressive accordance with data. However, there are at least three important aspects which put in evidence the incompleteness of the SM and, hence, the need for new physics. Namely they are (i) the non-zero neutrino masses, which allow for neutrino flavor oscillation during their space propagation, (ii) the lack of a consistent candidate for dark matter (DM), assuming that DM is a manifestation of an unknown particle, and (iii) the lack of a CP violating mechanism efficient enough to explain the observed matterantimatter asymmetry in the Universe.

\footnotetext{
a e-mail: brucesan@ift.unesp.br

b e-mail: ernany @th.physik.uni-bonn.de

${ }^{\mathrm{c}}$ e-mail: montero@ift.unesp.br
}

In this way, models based on the $\mathrm{SU}(3)_{C} \otimes \mathrm{SU}(3)_{L} \otimes$ $\mathrm{U}(1)_{N}$ gauge symmetry (the so-called 3-3-1 models, for shortness) [1-4], are interesting extensions of the SM. Since in these models the electroweak interaction is supposed to be invariant under transformations of a larger gauge group, the matter content can be chosen to accommodate new appropriate degrees of freedom in order to implement phenomenologically attractive features, as generation of neutrino masses, for instance.

The quark sector will also be enlarged and new quarks will be present, with the possibility of possessing exotic electric charges. Chiral anomaly cancellation is ensured provided we have the same number of triplets and anti-triplets, including color counting. If we assume that there is a symmetry between leptons and quarks in such a way that the number of families of leptons is equal to the number of families of quarks, say $N_{f}$, then we must have $N_{\text {anti }}=2 N_{f} / 3$ quark families transforming under the $\overline{\mathbf{3}}$ representation of the $\mathrm{SU}(3)_{L}$ group, and the other $N_{f}-N_{\text {anti }}$ families transforming under the 3 one. It means that the number of families must be three or a multiple of three. As a consequence, differently from the SM, the model is anomaly free only when the total number of families is considered.

Moreover, if we bring in the QCD asymptotic freedom, the number of families must be just 3 . The renormalization group $\beta$ function, which gives the behavior of the strong coupling with the transferred momentum, can be computed in perturbation theory and at one-loop level its sign is governed by the factor $-\left(11-\frac{2}{3} n_{q}\right)$, where $n_{q}$ is the number of quark flavors (which is 6 in the SM). To keep the negative sign, the only possibility compatible with asymptotic freedom, $n_{q}$ must be $\leq 16$. In 3-3-1 models with $N_{f}=3$ we have 9 quark flavors and the sign of the $\beta$ function remains correct. However, for $N_{f}=6$ there are 18 quark flavors and the $\beta$ function gets the wrong sign so that the number of families must be 3 . It is interesting to note that the anomaly cancellation relates the number of families to the number of colors. In this sense 
the 3-3-1 models shed some light on the family replication problem.

Besides this particular feature, 3-3-1 models are promising alternatives to the SM, for they present a variety of interesting properties. Among them we can mention: (i) in the model described in Ref. [1] we find that the $\mathrm{U}(1)_{N}$ and the $\mathrm{SU}(3)_{L}$ coupling constants, $g_{N}$ and $g_{L}$, respectively, obey the relation $t^{2}=\left(g_{N} / g_{L}\right)^{2}=\sin ^{2} \theta_{W} /\left(1-4 \sin ^{2} \theta_{W}\right)$, where $\theta_{W}$ is the electroweak Weinberg mixing angle. It means that there is a Landau-like pole at an $\mathcal{O}(\mathrm{TeV})$ energy scale $\mu$ such that $\sin ^{2} \theta_{W}(\mu)=1 / 4$ [5], and hence it explains why $\sin ^{2} \theta_{W}(\mu)<1 / 4$ is observed; (ii) the electric charge quantization is independent of the nature of neutrinos, i.e., regardless if they are Majorana or Dirac fermions [6]; (iii) the Peccei-Quinn symmetry, needed to solve the strong CP problem, is almost automatic in these models [7,8].

For each 3-3-1 model, depending on the matter content accommodated in the $\mathrm{SU}(3)_{L}$ triplets and singlets, an appropriate scalar sector has to be introduced. As usual, the Yukawa terms are responsible for generating mass to the matter fields and also for matter fields-(pseudo-)scalar interactions. Therefore, the 3-3-1 scalar sector is richer than that of the SM, and this fact can be explored to give explanations to some phenomenological aspects that do not have a consistent answer or are out of the scope of the SM framework. Among others, some aspects closely related to the scalar sector and the Yukawa interactions are (i) a mechanism for generating tiny neutrino masses [9-11], (ii) a natural explanation for the fermion mass hierarchy, and (iii) a consistent DM candidate [9, 12-15].

In order to achieve these goals, and recover the low energy physics, spontaneous symmetry breaking (SSB) must occur. For each linearly independent broken generator, there will be a Nambu-Goldstone (NG) boson, if the number of broken symmetries exceeds the number of massive gauge bosons, this implies physical massless NG bosons which are potentially dangerous since they couple to fermions and could in principle escape from star core carrying out energy, thus modifying the standard evolution of these objects. Their interactions with nucleons [16-22] and electrons [16,23-28], are parameterized by $g_{n n J}$ and $g_{e \bar{e} J}$, respectively, (where $J$ means the physical NG boson), are bounded by the standard evolution of neutron stars and supernovae; red giants, super giants, sun, white dwarfs and neutron star crusts, and can constrain the parameters of a given scenario or even rule it out. This is the main goal of this work.

This work is organized as follows. In Sect. 2, we present the general features of the 3-3-1 model, including its matter content, Yukawa and scalar potential. In Sect. 3, we consider the model with a $\mathbb{Z}_{2}$ symmetry widely used, and we present the consequences of this choice, such as the number and form of physical NG bosons for each configuration of vacuum expectation values (VEVs), and a discussion of the constraints from $g_{e \bar{e} J}$ and $Z$ invisible decay on them. Finally, in Sect. 4 , we add soft $\mathbb{Z}_{2}$-breaking terms to the previous scalar potential and analyze their consequences using as a guidance the constraints from the $g_{e \bar{e} J}$ and $g_{n n J}$ couplings and also the $W$ mass and the $\rho$ parameter values; a discussion of the constraints from a symmetry point of view is also presented. Section 5 is devoted to our conclusions.

\section{The Model}

The 3-3-1 model with right-handed neutrinos considered in this paper was proposed in Refs. [3,10] and it has been subsequently considered in Refs. [8,9,12,29-35], where different aspects of this model were studied. This model shares appealing features with other versions of 3-3-1 models [1-3,36-40]. Furthermore, right-handed neutrinos are in the same multiplet of the SM leptons, which allows for terms of mass for the neutrinos at tree level, although the smallness of the those masses remains unexplained.

Generally speaking, this model is based on the gauge symmetry group $\mathrm{SU}(3)_{C} \otimes \mathrm{SU}(3)_{L} \otimes \mathrm{U}(1)_{N}$, where $C$ stands for color and $L$ for left chirality, as in the SM; and $N$ stands for a new charge different from the SM hypercharge $Y$. The $N$ values are assigned in order to obtain the SM hypercharge $Y=2 N 1_{3 \times 3}-\frac{2}{\sqrt{3}} T_{8}$, after the first spontaneous symmetry breaking, and as a consequence the electric charge $Q=T_{3}+\frac{Y}{2} \mathbf{1}_{3 \times 3}$, where $T_{3}, T_{8}$ are the diagonal $\mathrm{SU}(3)_{L}$ generators, whereas $T_{9}\left(=\frac{N}{2} \mathbf{1}_{3 \times 3}\right)$ is the generator of the $\mathrm{U}(1)_{N}$ group. Symmetry breaking and fermion masses are achieved with at least three $\mathrm{SU}(3)_{L}$ triplets, $\eta, \rho, \chi$, as shown in Ref. [33]. These triplets are in the $(1, \mathbf{3},-1 / 3),(1, \mathbf{3}, 2 / 3)$ and $(1, \mathbf{3},-1 / 3)$ representations of the SU (3) $C \otimes S U(3)_{L} \otimes U(1)_{N}$ symmetry groups, respectively. In more detail, the scalar triplets are expressed by

$$
\begin{aligned}
& \eta=\left(\eta_{1}^{0}, \eta_{2}^{-}, \eta_{3}^{0}\right)^{\mathrm{T}}, \quad \rho=\left(\rho_{1}^{+}, \rho_{2}^{0}, \rho_{3}^{+}\right)^{\mathrm{T}}, \\
& \chi=\left(\chi_{1}^{0}, \chi_{2}^{-}, \chi_{3}^{0}\right)^{\mathrm{T}} .
\end{aligned}
$$

The fermionic content of the model is richer than the SM because the fields are embedded into non-trivial representations of a larger group, SU (3) ${ }_{L}$. The left-handed fields belong to the following representations:

$$
\text { Leptons: } \begin{aligned}
f_{a L} & =\left(\begin{array}{lll}
v_{a} & e_{a} & N_{a}^{c}
\end{array}\right)_{L}^{\mathrm{T}} \sim(1, \mathbf{3},-1 / 3), \\
\text { Quarks: } Q_{L} & =\left(\begin{array}{lll}
u_{1} & d_{1} & u_{4}
\end{array}\right)_{L}^{\mathrm{T}} \sim(\mathbf{3}, \mathbf{3}, 1 / 3), \\
Q_{b L} & =\left(\begin{array}{lll}
d_{b} & u_{b} & d_{b+2}
\end{array}\right)_{L}^{\mathrm{T}} \sim(\mathbf{3}, \overline{\mathbf{3}}, 0),
\end{aligned}
$$

where $a=1,2,3$ and $b=2,3$; and " " means the transformation properties under the local symmetry group. Notice that $N_{a}$ stands for the right-handed neutrinos. Additionally, in the right-handed field sector we have 
Leptons: $e_{a R} \sim(1,1,-1)$,

Quarks: $u_{s R} \sim(\mathbf{3}, 1,2 / 3), \quad d_{t R} \sim(3,1,-1 / 3)$,

with $a$ in the same range as in the previous case; $s=1, \ldots, 4$ and $t=1, \ldots, 5$.

Regarding the Yukawa Lagrangian, we can write it as follows:

$\mathcal{L}_{\text {Yuk }}=\mathcal{L}_{\text {Yuk }}^{\rho}+\mathcal{L}_{\text {Yuk }}^{\eta}+\mathcal{L}_{\text {Yuk }}^{\chi}$,

with

$$
\begin{aligned}
\mathcal{L}_{\text {Yuk }}^{\rho}= & \alpha_{t} \bar{Q}_{L} d_{t R} \rho+\alpha_{b s} \bar{Q}_{b L} u_{s R} \rho^{*} \\
& +\mathrm{Y}_{a a^{\prime}} \varepsilon_{i j k}\left(\bar{f}_{a L}\right)_{i}\left(f_{a^{\prime} L}\right)_{j}^{c}\left(\rho^{*}\right)_{k} \\
& +\mathrm{Y}_{a a^{\prime}}^{\prime} \bar{f}_{a L} e_{a^{\prime} R} \rho+\text { H.c., } \\
\mathcal{L}_{\text {Yuk }}^{\eta}= & \beta_{s} \bar{Q}_{L} u_{s R} \eta+\beta_{b t} \bar{Q}_{b L} d_{t R} \eta^{*}+\text { H.c., } \\
\mathcal{L}_{\text {Yuk }}^{\chi}= & \gamma_{s} \bar{Q}_{L} u_{s R} \chi+\gamma_{b t} \bar{Q}_{b L} d_{t R} \chi^{*}+\text { H.c. },
\end{aligned}
$$

where $a^{\prime}, i, j, k=1,2,3$ and with $a, b, s, t$ in the same range as in the previous case. From Eqs. (5)-(7) it can be seen that, in general, flavor-changing neutral currents (FCNCs) can be induced because the quark fields interact with different neutral scalar fields simultaneously. This characteristic is shared by most of multi-Higgs models [34,41]. However, some model dependent strategies to successfully overcome that problem have been proposed [3,41,42]. Among those, we can mention, for instance, choosing an appropriate direction in the VEV space, resorting to heavy scalars and/or small mixing angles in the quark and the scalar sectors, and considering adequate Yukawa coupling matrix textures. In particular, in this model the non-SM quarks have the same electric charge as the SM ones. That means that these can mix with the latter ones and hence also induce FCNC. Despite that, this kind of FCNC is suppressed when the VEV which mainly controls the exotic quark masses is taken much larger than the electroweak mass scale [42]. Also, FCNC occurs in models which have an extra neutral vector boson. These can be handled in a similar way. See, for example, $[43,44]$. Finally, we remark that from Eq. (5) it is clear that the lepton sector of the model is not afflicted by FCNC.

The most general scalar potential consistent with gauge invariance and renormalizability is given by

$V(\eta, \rho, \chi)=V_{\mathbb{Z}_{2}}(\eta, \rho, \chi)+V_{\mathbb{Z} / 2}(\eta, \rho, \chi) ;$

with

$$
\begin{aligned}
V_{\mathbb{Z}_{2}}(\eta, \rho, \chi)= & -\mu_{1}^{2} \eta^{\dagger} \eta-\mu_{2}^{2} \rho^{\dagger} \rho-\mu_{3}^{2} \chi^{\dagger} \chi \\
& +\lambda_{1}\left(\eta^{\dagger} \eta\right)^{2}+\lambda_{2}\left(\rho^{\dagger} \rho\right)^{2}+\lambda_{3}\left(\chi^{\dagger} \chi\right)^{2} \\
& +\lambda_{4}\left(\chi^{\dagger} \chi\right)\left(\eta^{\dagger} \eta\right) \\
& +\lambda_{5}\left(\chi^{\dagger} \chi\right)\left(\rho^{\dagger} \rho\right)+\lambda_{6}\left(\eta^{\dagger} \eta\right)\left(\rho^{\dagger} \rho\right)
\end{aligned}
$$

$$
\begin{aligned}
& +\lambda_{7}\left(\chi^{\dagger} \eta\right)\left(\eta^{\dagger} \chi\right) \\
& +\lambda_{8}\left(\chi^{\dagger} \rho\right)\left(\rho^{\dagger} \chi\right)+\lambda_{9}\left(\eta^{\dagger} \rho\right)\left(\rho^{\dagger} \eta\right) \\
& +\left[\lambda_{10}\left(\chi^{\dagger} \eta\right)^{2}+\text { H.c. }\right]
\end{aligned}
$$

$$
\begin{aligned}
V_{\mathbb{Z}_{2}}(\eta, \rho, \chi)= & -\mu_{4}^{2} \chi^{\dagger} \eta \\
& +\lambda_{11}\left(\chi^{\dagger} \eta\right)\left(\eta^{\dagger} \eta\right)+\lambda_{12}\left(\chi^{\dagger} \eta\right)\left(\chi^{\dagger} \chi\right) \\
& +\lambda_{13}\left(\chi^{\dagger} \eta\right)\left(\rho^{\dagger} \rho\right) \\
& +\lambda_{14}\left(\chi^{\dagger} \rho\right)\left(\rho^{\dagger} \eta\right) \\
& +\frac{f}{\sqrt{2}} \epsilon_{i j k} \eta_{i} \rho_{j} \chi_{k}+\text { H.c. }
\end{aligned}
$$

We have divided the total scalar potential $V(\eta, \rho, \chi)$ in two pieces, $V_{\mathbb{Z}_{2}}(\eta, \rho, \chi)$ and $V_{\mathbb{Z} Z}(\eta, \rho, \chi)$, for future convenience. The first part is invariant under a $\mathbb{Z}_{2}$ discrete symmetry $\left(\chi \rightarrow-\chi, u_{4 R} \rightarrow-u_{4 R}, d_{(4,5) R} \rightarrow-d_{(4,5) R}\right)$ in contrast to the second one which is not. The model with such $\mathbb{Z}_{2}$ symmetry will be studied in detail in the next section.

The minimal vacuum structure in order to give masses for all the fields in the model is

$$
\begin{aligned}
& \langle\rho\rangle=\frac{1}{\sqrt{2}}\left(\begin{array}{lll}
0 & v_{\rho_{2}} & 0
\end{array}\right)^{\mathrm{T}}, \quad\langle\eta\rangle=\frac{1}{\sqrt{2}}\left(\begin{array}{llll}
v_{\eta_{1}} & 0 & 0
\end{array}\right)^{\mathrm{T}}, \\
& \langle\chi\rangle=\frac{1}{\sqrt{2}}\left(\begin{array}{lll}
0 & 0 & v_{\chi_{3}}
\end{array}\right)^{\mathrm{T}} .
\end{aligned}
$$

Specifically, the symmetry breaking pattern is done in two stages. First, when $\chi$ gains a VEV, $\langle\chi\rangle$, the exotic quarks gain masses and the symmetry, $\mathrm{SU}(3)_{C} \otimes \mathrm{SU}(3)_{L} \otimes \mathrm{U}(1)_{N}$, is broken down to $\mathrm{SU}(3)_{C} \otimes \mathrm{SU}(2)_{L} \otimes \mathrm{U}(1)_{Y}$. After that, the $\mathrm{VEV}\langle\rho\rangle$ gives mass to the three charged leptons and to two of the neutrinos $[4,10,11]$. Also, two up-type quarks and one down-type quark gain masses from $\langle\rho\rangle$. Finally, $\langle\eta\rangle$ gives mass for the remaining quarks. In this last stage $(\langle\rho\rangle$ and $\langle\eta\rangle$ different from zero) the symmetry, $\mathrm{SU}(3)_{C} \otimes \mathrm{SU}(2)_{L} \otimes \mathrm{U}(1)_{Y}$, is broken down to $\mathrm{U}(1)_{Q}$. Note that, although the $\eta$ and $\chi$ scalar triplets are in the same representation of the gauge symmetries, we have defined, without loss of generality, the $\chi$ triplet as the one responsible for the first symmetry breaking stage. In other words, it is assumed that $\langle\chi\rangle>\langle\rho\rangle,\langle\eta\rangle$.

\section{Model with $\mathbb{Z}_{2}$ symmetry}

It is a common practice to impose the discrete $\mathbb{Z}_{2}$ symmetry given by $\chi \rightarrow-\chi, u_{4 R} \rightarrow-u_{4 R}, d_{(4,5) R} \rightarrow$ $-d_{(4,5) R}$ and all the other fields being even under $\mathbb{Z}_{2}[4,8-$ $10,12,30,31,45]$. This symmetry brings simplicity to the 
model allowing, for instance, to interpret the $\chi$ scalar as the responsible for the first step in the symmetry breaking pattern and in some sense, to mitigate the FCNC issues. There are, in principle, additional reasons to consider the model with the exact $\mathbb{Z}_{2}$ symmetry. Among those reasons, we can mention, for example, the stabilization of dark matter candidates $[9,12]$ and the naturalness of the implementation of the PQ mechanism [8]. In this scenario, the Yukawa Lagrangian interactions given in Eqs. (5)-(7) are slightly modified to

$$
\begin{aligned}
\mathcal{L}_{\text {Yuk }}^{\rho}= & \alpha_{a} \bar{Q}_{L} d_{a R} \rho+\alpha_{b a} \bar{Q}_{b L} u_{a R} \rho^{*} \\
& +\mathrm{Y}_{a a^{\prime}} \varepsilon_{i j k}\left(\bar{f}_{a L}\right)_{i}\left(f_{b L}\right)_{j}^{c}\left(\rho^{*}\right)_{k} \\
& +\mathrm{Y}_{a a^{\prime}}^{\prime} \bar{f}_{a L} e_{a^{\prime} R} \rho+\text { H.c. }, \\
\mathcal{L}_{\text {Yuk }}^{\eta}= & \beta_{a} \bar{Q}_{L} u_{a R} \eta+\beta_{b a} \bar{Q}_{b L} d_{a R} \eta^{*}+\text { H.c. }, \\
\mathcal{L}_{\text {Yuk }}^{\chi}= & \gamma_{4} \bar{Q}_{L} u_{4 R} \chi+\gamma_{b(b+2)} \bar{Q}_{b L} d_{(b+2) R} \chi^{*}+\text { H.c. . }
\end{aligned}
$$

Furthermore, the $\mathbb{Z}_{2}$ symmetry forbids the terms in $V_{\mathbb{Z} / 2}(\eta, \rho, \chi)$ to appear in the scalar potential. It implies that the model has actually a larger symmetry group. Specifically, we show in Table 1 all the U(1) symmetries that the model really has (the global and local ones). Note there are two extra global symmetries, the baryonic ones, $\mathrm{U}(1)_{B}$, that remain unbroken, and the $\mathrm{U}(1)_{\mathrm{PQ}}$. The last one is a PecceiQuinn like symmetry because it is anomalous in the color group. We also remark that these are symmetries of the entire Lagrangian.

In the minimal case, when only three VEVs $-v_{\eta_{1}}, v_{\rho_{2}}$ and $v_{\chi_{3}}$-are different from zero, the scalar sector has a NambuGoldstone (NG) boson, $J$, in the physical spectrum. It is given by

$J=\frac{1}{N_{J}}\left(\frac{v_{\eta_{1}} v_{\chi_{3}}}{v_{\rho_{2}}} \operatorname{Im} \rho_{2}^{0}+v_{\chi_{3}} \operatorname{Im} \eta_{1}^{0}+v_{\eta_{1}} \operatorname{Im} \chi_{3}^{0}\right)$,

with $N_{J} \equiv\left(v_{\eta_{1}}^{2} v_{\chi_{3}}^{2} v_{\rho_{2}}^{-2}+v_{\eta_{1}}^{2}+v_{\chi_{3}}^{2}\right)^{1 / 2}$. We emphasize that $J$ in Eq. (14) is orthogonal to the NG bosons which are absorbed by the gauge vector bosons, as it should be. We have followed the method described in Refs. [46,47] to accomplish that.

From the explicit form of $J$ in Eq. (14), and the Lagrangian in Eq. (11), it is straightforward to calculate the coupling of $J$ with the electron and positron, $g_{e \bar{e} J}$. It is explicitly given by

$g_{e \bar{e} J}=\frac{\sqrt{2} m_{e} v_{\eta_{1}} v_{\chi_{3}}}{N_{J} v_{\rho_{2}}^{2}}$

where $m_{e}$ is the electron mass. The existence of this coupling opens a new channel of energy loss in stars through the Compton-type process $\gamma+e^{-} \rightarrow e^{-}+J$. From the evolution of red-giant stars we have [16,23-26]

$\left|g_{\text {eê } J}\right| \lesssim g_{\max } \equiv 10^{-13}$.
In order to impose a bound on the VEVs, we bring in another piece of information. The mass of the $W^{ \pm}$bosons is

$M_{W^{ \pm}}^{2}=\frac{g_{L}^{2}}{4}\left(v_{\eta_{1}}^{2}+v_{\rho_{2}}^{2}\right)$,

where $g_{L}$ is the gauge coupling constant of the $\mathrm{SU}(3)_{L}$ group. Thus, $v_{\eta_{1}}^{2}+v_{\rho_{2}}^{2}=v_{\mathrm{SM}}^{2} \simeq 246^{2} \mathrm{GeV}^{2}$, where $v_{\mathrm{SM}}$ is the symmetry breaking energy scale of the SM in order to obtain the SM $W^{ \pm}$mass [48]. From Eqs. (15)-(17) the following upper bound on $v_{\chi_{3}}$ can be found:

$$
\begin{aligned}
v_{\chi_{3}} & \leq v_{\chi \max }\left(v_{\rho_{2}}\right) \\
& \equiv v_{\rho_{2}}\left[2 g_{\max }^{-2} m_{e}^{2} / v_{\rho_{2}}^{2}-1 /\left(1-v_{\rho_{2}}^{2} / v_{\mathrm{SM}}^{2}\right)\right]^{-1 / 2} .
\end{aligned}
$$

Thus, the upper bound on $v_{\chi_{3}}$ is a function of $v_{\rho_{2}}$. Because of Eq. (18), we can estimate that the largest value that $v_{\chi_{3}}$ can take is $v_{\chi \max }\left(v_{\rho_{2}} \rightarrow v_{\mathrm{SM}}\right) \simeq 11.5 \mathrm{keV}$. However, it is in contradiction with the general assumption in this model which claims that $\langle\chi\rangle>\langle\rho\rangle,\langle\eta\rangle$. In addition to that, there is a fact that rules out this scenario with the $\mathbb{Z}_{2}$ symmetry. In order to understand it, notice that the NG boson in Eq. (14), which results from the breaking of the symmetries, is actually an axion because of the $\mathrm{U}(1)_{\mathrm{PQ}}$ symmetry in Table 1 . Moreover, the decay constant $f_{a}$ for the axion is, in this case, given by

$f_{a}=N_{J}$.

From this and the upper bound in Eq. (18) we can find an upper bound on $f_{a}$ as follows:

$f_{a} \leq f_{a \max }\left(v_{\rho_{2}}\right) \equiv v_{\mathrm{SM}}\left[1-\frac{v_{\rho_{2}}^{2}}{v_{\mathrm{SM}}^{2}}+\frac{v_{\chi \max }^{2}\left(v_{\rho_{2}}\right)}{v_{\rho_{2}}^{2}}\right]^{1 / 2}$.

From the equation above, we can see that $v_{\chi \max }\left(v_{\mathrm{SM}}\right) \approx$ $11.5 \mathrm{keV} \leq f_{a} \leq v_{\mathrm{SM}}$. However, an axion with this small decay constant was ruled out long ago $[49,50]$.

In the symmetry breaking patterns with more than three VEVs different from zero, i.e., $\left(v_{\eta_{1}}, v_{\eta_{3}}, v_{\rho_{2}}, v_{\chi_{3}}\right),\left(v_{\eta_{1}}, v_{\rho_{2}}\right.$, $\left.v_{\chi_{1}}, v_{\chi_{3}}\right)$, and $\left(v_{\eta_{1}}, v_{\eta_{3}}, v_{\rho_{2}}, v_{\chi_{1}}, v_{\chi_{3}}\right)$, the situation is even worse. Although the exact forms of the CP-even and CPodd scalars present in the physical spectra depend on the particular breaking pattern of the symmetry, there are some general features that we can mention. In all cases, there are two physical NG bosons, $J_{I}$ and $J_{R}$, the first in the CP-odd sector, and the second in the CP-even sector. One of them couples with the electron and positron, i.e., $g_{e \bar{e} J_{I}} \neq 0$, which imposes upper bounds on the VEVs as in the previous case. Moreover, the existence of $J_{R}$ in the physical spectrum brings about an extra difficulty that rules out the model once and for all when that $\mathbb{Z}_{2}$ symmetry is considered. This difficulty comes from the invisible decay width of the $Z$ gauge boson, 
Table 1 The U(1) symmetries in the model when the $\mathbb{Z}_{2}$ discrete symmetry is considered

\begin{tabular}{llllllllll}
\hline & $Q_{L}$ & $Q_{i L}$ & $\left(u_{a R}, u_{4 R}\right)$ & $\left(d_{a R}, d_{(4,5) R}\right)$ & $f_{a L}$ & $e_{a R}$ & $\eta$ & $\rho$ & $\chi$ \\
\hline $\mathrm{U}(1)_{N}$ & $1 / 3$ & 0 & $2 / 3$ & $-1 / 3$ & $-1 / 3$ & -1 & $-1 / 3$ & $2 / 3$ & $-1 / 3$ \\
$\mathrm{U}(1)_{B}$ & $1 / 3$ & $1 / 3$ & $1 / 3$ & $1 / 3$ & 0 & 0 & 0 & 0 & 0 \\
$\mathrm{U}(1)_{\mathrm{PQ}}$ & 1 & -1 & 0 & 0 & $-1 / 2$ & $-3 / 2$ & 1 & 1 & 1 \\
\hline
\end{tabular}

which receives at least one new contribution, $Z \rightarrow J_{R} J_{I}$, and there is no more room for this [48]. Therefore, we can conclude that the model invariant under the $\mathbb{Z}_{2}$ symmetry is not consistent with constraints coming from astrophysics and particle physics.

\section{Model with soft $\mathbb{Z}_{2}$-breaking terms}

There is another possibility to be taken into account when the $\mathbb{Z}_{2}$ discrete symmetry is considered. This is to introduce some soft $\mathbb{Z}_{2}$-breaking terms in the scalar potential in order to remove the extra accidental symmetries in the Lagrangian, and at the same time leave the model as simple as possible. Keeping that in mind, we are going to explore the only two soft $\mathbb{Z}_{2}$ violating terms (i.e., operators with mass dimension less than 4 that break the $\mathbb{Z}_{2}$ symmetry), $\mu_{4}^{2} \chi^{\dagger} \eta$ and $\frac{f}{\sqrt{2}} \epsilon_{i j k} \eta_{i} \rho_{j} \chi_{k}$, that can in principle accomplish that. In other words, we are seeking for some strategy in order to recover the safety of the model. Note that, in contrast to considering explicit symmetry breaking terms having dimension $\geq 4$, the advantage of considering soft terms is that radiative corrections cannot destabilize any result derived from symmetry arguments cf. [51,52].

\section{$4.1 \mu_{4}^{2} \chi^{\dagger} \eta$ term}

First, we consider the $\mu_{4}^{2} \chi^{\dagger} \eta$ soft-breaking term together with the scalar potential allowed by the $\mathbb{Z}_{2}$ symmetry. Although this term does not remove any extra symmetry in Table 1, its presence slightly changes the form of the NG boson in comparison to the case of the model without it. In some sense that justifies our next analysis. In order to extract conclusions we obtain the physical NG boson for each symmetry breaking pattern. The NG boson looks in each case as follows:

$$
\begin{aligned}
& v_{\eta_{1}}, v_{\rho_{2}}, v_{\chi_{3}} \rightarrow J_{1}=N_{J_{1}}^{-1}\left(v_{\chi_{3}} \operatorname{Im} \eta_{1}^{0}+\frac{v_{\eta_{1}} v_{\chi_{3}}}{v_{\rho_{2}}} \operatorname{Im} \rho_{2}^{0}\right. \\
& \left.+v_{\eta_{1}} \operatorname{Im} \chi_{3}^{0}\right) \\
& v_{\eta_{1}}, v_{\eta_{3}}, v_{\rho_{2}}, v_{\chi_{3}} \rightarrow J_{2}=N_{J_{2}}^{-1}\left(v_{\chi_{3}} \operatorname{Im} \eta_{1}^{0}+\frac{v_{\eta_{1}} v_{\chi_{3}}}{v_{\rho_{2}}} \operatorname{Im} \rho_{2}^{0}\right. \\
& \left.+v_{\eta_{1}} \operatorname{Im} \chi_{3}^{0}-v_{\eta_{3}} \operatorname{Im} \chi_{1}^{0}\right)
\end{aligned}
$$

$$
\begin{aligned}
& v_{\eta_{1}}, v_{\rho_{2}}, v_{\chi_{1}}, v_{\chi_{3}} \rightarrow J_{3}=N_{J_{3}}^{-1}\left(v_{\chi_{3}} \operatorname{Im} \eta_{1}^{0}+\frac{v_{\eta_{1}} v_{\chi_{3}}}{v_{\rho_{2}}} \operatorname{Im} \rho_{2}^{0}\right. \\
& \left.+v_{\eta_{1}} \operatorname{Im} \chi_{3}^{0}-v_{\chi_{1}} \operatorname{Im} \eta_{3}^{0}\right),
\end{aligned}
$$

$v_{\eta_{1}}, v_{\eta_{3}}, v_{\rho_{2}}, v_{\chi_{1}}, v_{\chi_{3}} \rightarrow J_{4}=N_{J_{4}}^{-1}\left(v_{\chi_{3}} \operatorname{Im} \eta_{1}^{0}\right.$

$$
-\frac{\left(v_{\eta_{3}} v_{\chi_{1}}-v_{\eta_{1}} v_{\chi_{3}}\right)}{v_{\rho_{2}}} \operatorname{Im} \rho_{2}^{0}+v_{\eta_{1}} \operatorname{Im} \chi_{3}^{0}-v_{\chi_{1}} \operatorname{Im} \eta_{3}^{0}
$$$$
\left.-v_{\eta_{3}} \operatorname{Im} \chi_{1}^{0}\right) \text {, }
$$

where $N_{J_{i}}$ (normalization factors) are given by $N_{J_{i}} \equiv$ $\left(C_{\eta_{1}}^{2}+C_{\eta_{3}}^{2}+C_{\rho_{2}}^{2}+C_{\chi_{1}}^{2}+C_{\chi_{3}}^{2}\right)^{1 / 2}$, and we also have defined

$$
\begin{aligned}
J_{i} \equiv & N_{J_{i}}^{-1}\left[C_{\rho_{2}} \operatorname{Im} \rho_{2}^{0}+C_{\eta_{1}} \operatorname{Im} \eta_{1}^{0}+C_{\eta_{3}} \operatorname{Im} \eta_{3}^{0}\right. \\
& \left.+C_{\chi_{1}} \operatorname{Im} \chi_{1}^{0}+C_{\chi_{3}} \operatorname{Im} \chi_{3}^{0}\right] .
\end{aligned}
$$

One can note that the component $\operatorname{Im} \rho_{2}^{0}$ in $J$ accounts for a non-zero value of $g_{e \bar{e} J}$, which is generically given by $g_{e \bar{e} J_{i}}=\frac{\sqrt{2} m_{e}}{v_{\rho_{2}}} N_{J_{i}}^{-1} C_{\rho_{2}}$. The case $\left(v_{\eta_{1}}, v_{\rho_{2}}, v_{\chi_{3}}\right)$ is ruled out following discussion after Eq. (18), since we exactly recover the case of Eqs. (14) and (15). For the cases with more than three non-vanishing VEVs, one has to deal with more variables which makes these three scenarios- $J_{2}, J_{3}$ and $J_{4}$-not easy to exclude using the same strategy followed in Sect. 3. The best we can do with that strategy is set an upper bound on $v_{\chi_{3}}$. For the case of four VEVs we manage to find $v_{\chi_{3}} \lesssim 355 \mathrm{GeV}$. The case of the five VEVs is more intricate although a similar bound (although not general) is possible to get. Roughly speaking, these bounds come from the simultaneous application of the $g_{e \bar{e} J}$ and $\rho=M_{W}^{2} /\left(M_{Z}^{2} \cos ^{2} \theta_{W}\right)$ bounds, where $M_{Z}$ is the $Z$ boson mass. There is another immediate consequence arising from the $J$ form. Following Eq. (4), and taking into account the expressions of the $J$ NG bosons in Eqs. (21)-(24), we are led to the conclusion that they are going to interact inevitably with the quarks of the model. It implies that the $g_{n n J}$ coupling, the interaction of two nucleons with $J$, is different from zero in this case. Nevertheless, it must be smaller than $10^{-12}$, i.e., $\left|g_{n n J}\right| \lesssim g_{n n J}^{\max } \equiv 10^{-12}[16-22]$. We have checked that this bound eventually imposes strong constraints on the VEVs and the Yukawa couplings of the model. In general, we have seen that some parameters are forced to have an $\mathcal{O}\left(10^{-15}\right)$ 
tuning in order for the limit on $g_{n n J}$ to be respected. However, we are not going into the details because there is a different line of reasoning that unquestionably rules out this scenario with just the $\mu_{4}^{2} \chi^{\dagger} \eta$ term. It is based on the observation that the $\mathrm{U}(1)_{\mathrm{PQ}}$ which generates the $\mathrm{NG}$ boson (in this case it is an axion) is actually broken at the electroweak scale. In order to see that, note that the $3 \mathrm{U}(1)_{N}+\mathrm{U}(1)_{\mathrm{PQ}}$ subgroup is not spontaneously broken by any $\eta_{i}$ or $\chi_{i}$ VEVs. Thus, the responsible for breaking that subgroup is the $\rho$ VEV Thus, the VEV responsible for breaking that subgroup is the $\rho \mathrm{VEV}$, which is upper-bounded by the electroweak scale $\left(v_{\mathrm{SM}} \simeq 246 \mathrm{GeV}\right)$ since it is mainly responsible for giving mass to all SM leptons in this model. Therefore, the NG boson (axion) would be visible and thus already ruled out. All in all, the soft $\mu_{4}^{2} \chi^{\dagger} \eta$ breaking term is not capable to get rid of the issues arising from the presence of a NG boson in this model.

\section{$4.2 \frac{f}{\sqrt{2}} \epsilon_{i j k} \eta_{i} \rho_{j} \chi_{k}$ term}

From Table 1 , we can see that the $\frac{f}{\sqrt{2}} \epsilon_{i j k} \eta_{i} \rho_{j} \chi_{k}$ term removes the $\mathrm{U}(1)_{\mathrm{PQ}}$ symmetry [34]. As a result there are no physical NG bosons, and that leaves the model safe regarding the appearance of these massless particles. Therefore, we conclude that this model can be considered with the $\mathbb{Z}_{2}$ symmetry provided the soft $\frac{f}{\sqrt{2}} \epsilon_{i j k} \eta_{i} \rho_{j} \chi_{k}$ term is included in the scalar potential. Needless to say, the necessity of keeping this softbreaking term brings about some consequences: for instance, if the $\mathbb{Z}_{2}$ symmetry is used to stabilize a dark matter candidate, say the $\chi_{1}^{0}$ scalar, the coupling $f$ must be extremely suppressed in order to guarantee the stability of the DM candidate on a cosmological time scale. Moreover, in a version of the 3-3-1 model with exotic leptons, this trilinear term controls the exotic lepton mean lifetime [53].

\section{Conclusions}

The presence of physical NG bosons plays a potentially important role in constraining the parameters of a model, and in some cases excludes it. The reason is that any light neutral particle can interact with matter providing an important stellar energy-loss mechanism. With this motivation, we have constrained an appealing version of the 3-3-1 model, taking into account the presence of physical NG bosons, which are inconsistent with both astrophysical and particle physics well established results. Specifically, we have considered the 3-3-1 model with right-handed neutrinos when a $\mathbb{Z}_{2}$ symmetry is imposed. This scenario is conventionally considered in the literature because it greatly simplifies the model. However, that discrete symmetry brings as a consequence the introduction about of an extra global sym- metry, $\mathrm{U}(1)_{\mathrm{PQ}}$, which when spontaneously broken by the $v_{\eta_{1}}, v_{\rho_{2}}, v_{\chi_{3}}$ VEVs, introduces an axion in the model. The issue is that imposing simultaneously the bounds on $g_{e \bar{e} J}$ and $M_{W}$ we found bounds on the decay coupling constant $f_{a}$, specifically, $11.5 \mathrm{keV} \leq f_{a} \leq v_{\mathrm{SM}}$. It is inconsistent with experiments looking for light scalars (or pseudo-scalars) since that window for $f_{a}$ makes the axion visible. In addition to that, we have considered all the other possibilities for the available VEVs in the model, showing that these are also excluded. The reason is that the appearance of physical NG bosons contributing to the invisible decay width of the $Z$ gauge boson $\left(Z \rightarrow J_{R} J_{I}\right)$ is in conflict with experimental data. All of these scenarios are then ruled out.

We also studied in detail the soft $\mathbb{Z}_{2}$-symmetry breaking case. The two terms allowed by the gauge symmetries are $\mu_{4}^{2} \chi^{\dagger} \eta$ and $\frac{f}{\sqrt{2}} \epsilon_{i j k} \eta_{i} \rho_{j} \chi_{k}$. In the first case, we found that although the NG bosons have slightly different forms, there are also problems that exclude all of those scenarios. The main reason is that a $3 \mathrm{U}(1)_{N}+\mathrm{U}(1)_{\mathrm{PQ}}$ subgroup remains unbroken after the first step of the spontaneous symmetry breaking. Thus, the decay coupling constant is of the order of the electroweak scale, $v_{\mathrm{SM}}$, implying the same issues as the previous case. Finally, we show that the model can be considered consistent with the $\mathbb{Z}_{2}$ symmetry when the $\frac{f}{\sqrt{2}} \epsilon_{i j k} \eta_{i} \rho_{j} \chi_{k}$ term is included in the scalar potential. That term breaks the $\mathbb{Z}_{2}$ symmetry softly and removes the extra global symmetry. Thus, no physical NG boson appears at all and the model is safe concerning this issue.

Acknowledgements B. L. S. V. would like to thank Coordenação de Aperfeiçoamento de Pessoal de Nível Superior (CAPES), Brazil, for financial support. E. R. S. would like to thank Conselho Nacional de Desenvolvimento Científico e Tecnológico (CNPq), Brazil, for financial support and Bonn University for kind hospitality.

Open Access This article is distributed under the terms of the Creative Commons Attribution 4.0 International License (http://creativecomm ons.org/licenses/by/4.0/), which permits unrestricted use, distribution, and reproduction in any medium, provided you give appropriate credit to the original author(s) and the source, provide a link to the Creative Commons license, and indicate if changes were made. Funded by $\mathrm{SCOAP}^{3}$.

\section{References}

1. F. Pisano, V. Pleitez, Phys. Rev. D 46, 410 (1992)

2. P.H. Frampton, Phys. Rev. Lett. 69, 2889 (1992)

3. J.C. Montero, F. Pisano, V. Pleitez, Phys. Rev. D 47, 2918 (1993)

4. R. Foot, O.F. Hernández, F. Pisano, V. Pleitez, Phys. Rev. D 47, 4158 (1993)

5. A.G. Dias, R. Martinez, V. Pleitez, Eur. Phys. J. C 39, 101 (2005)

6. C.A.S. de Pires, O.P. Ravinez, Phys. Rev. D 58, 035008 (1998)

7. Alex G. Dias, V. Pleitez, Phys. Rev. D 69, 077702 (2004)

8. J.C. Montero, B.L. Sánchez-Vega, Phys. Rev. D 84, 055019 (2011)

9. J.K. Mizukoshi, C.A.S. de Pires, F.S. Queiroz, P.S.R. da Silva, Phys. Rev. D 83, 065024 (2011) 
10. R. Foot, H.N. Long, T.A. Tran, Phys. Rev. D 50, R34 (1994)

11. M.B. Tully, G.C. Joshi, Phys. Rev. D 64, 011301 (2001)

12. D. Cogollo, A.X. Gonzalez-Morales, F.S. Queiroz, P.R. Teles, J. Cosmol. Astropart. Phys. JCAP 1411, 002 (2014)

13. D. Fregolente, M.D. Tonasse, Phys. Lett. B 555, 7 (2003)

14. H.N. Long, N.Q. Lan, Europhys. Lett. 64, 571 (2003)

15. J.C. Montero, A. Romero, B.L. Sánchez-Vega, arXiv:1709.04535 [hep-ph]

16. H. Cheng, Phys. Rev. D 36, 1649 (1987)

17. G.G. Raffelt, in Axions: Theory, Cosmology and Experimental Searches, ed. by M. Kuster, G. Raffelt, B. Beltrán (Springer, Berlin, 2008), pp. 51-71

18. A. Burrows, M.S. Turner, R.P. Brinkmann, Phys. Rev. D 39, 1020 (1989)

19. A. Burrows, M.T. Ressell, M.S. Turner, Phys. Rev. D 42, 3297 (1990)

20. G.G. Raffelt, Stars as laboratories for fundamental physics (Chicago Univ. Pr, Chicago, 1996)

21. N. Iwamoto, Phys. Rev. Lett. 53, 1198 (1984)

22. A. Pantziris, K. Kang, Phys. Rev. D 33, 3509 (1986)

23. G. Raffelt, A. Weiss, Phys. Rev. D 51, 1495 (1995)

24. M. Fukugita, S. Watamura, M. Yoshimura, Phys. Rev. D 26, 1840 (1982)

25. G.G. Raffelt, Phys. Rev. D 33, 897 (1986)

26. D.S.P. Dearborn, D.N. Schramm, G. Steigman, Phys. Rev. Lett. 56, $26(1986)$

27. C.D.R. Carvajal, B.L. Sánchez-Vega, O. Zapata, Phys. Rev. D 96, 115035 (2017)

28. C.D.R. Carvajal, A.G. Dias, C.C. Nishi, B.L. Sánchez-Vega, JHEP 1505, 069 (2015)

29. C.A.S. de Pires, P.S.R. da Silva, Eur. Phys. J. C 36, 397 (2004)

30. A.G. Dias, C.A.S. de Pires, P.S.R. da Silva, Phys. Lett. B 628, 85 (2005)
31. P.V. Dong, Tr.T. Huong, D.T. Huong, H.N. Long, Phys. Rev. D 74, 053003 (2006)

32. P.V. Dong, H.N. Long, D.V. Soa, Phys. Rev. D 75, 073006 (2007)

33. J.C. Montero, B.L. Sánchez-Vega, Phys. Rev. D 91, 037302 (2015)

34. S.M. Boucenna, J.W.F. Valle, A. Vicente, Phys. Rev. D 92, 053001 (2015)

35. S. Profumo, F.S. Queiroz, Eur. Phys. J. C 74, 2960 (2014)

36. L. Clavelli, T.C. Yang, Phys. Rev. D 10, 658 (1974)

37. B.W. Lee, S. Weinberg, Phys. Rev. Lett. 38, 1237 (1977)

38. B.W. Lee, R.E. Shrock, Phys. Rev. D 17, 2410 (1978)

39. M. Singer, Phys. Rev. D 19, 296 (1979)

40. M. Singer, J.W.F. Valle, J. Schechter, Phys. Rev. D 22, 738 (1980)

41. T.P. Cheng, Marc Sher, Phys. Rev. D 35, 3484 (1987)

42. P. Langacker, D. London, Phys. Rev. D 38, 886 (1988)

43. R.H. Benavides, Y. Giraldo, W.A. Ponce, Phys. Rev. D 80, 113009 (2009)

44. A.C.B. Machado, J.C. Montero, V. Pleitez, Phys. Rev. D 88, 113002 (2013)

45. A.G. Dias, C.A.S. de Pires, P.S.R. da Silva, Phys. Rev. D 68, 115009 (2003)

46. M. Srednicki, Nucl. Phys. B 260, 011301 (1985)

47. M. Srednicki, Quantum Field Theory (Cambridge Univ. Pr, New York, 2007)

48. C. Patrignani et al. (Particle Data Group), Chin. Phys. C 40, 100001 (2016)

49. J. Preskill, M.B. Wise, F. Wilczek, Phys. Lett. B 120, 127 (1983)

50. R.D. Peccei, J. Korean Phys. Soc. 29, S199 (1996). arXiv:hep-ph/9606475

51. S. Dimopoulos, H. Georgi, Nucl. Phys. B 193, 150 (1981)

52. S.P. Martin, arXiv:hep-ph/9709356

53. G. De Conto, V. Pleitez, JHEP 1705, 104 (2017) 